\title{
Manejo conservador de la ruptura prematura de membranas pretérmino
}

\author{
María Cristina Barco Burgos*; Edgar Adel Noreña Mósquera**; Óscar de Jesús Ocampo Duque**; \\ Edwin Rafael Salcedo Corena**
}

\section{RESUMEN}

Se presenta un estudio descriptivo prospectivo en 52 mujeres con diagnóstico de ruptura de membranas entre las 26 y 35 semanas de gestación, a las cuales se les realizó un manejo conservador. Se controlaron con parámetros clínicos para corioamnionitis y paraclínicos como: leucocitosis mayor de $\mathbf{2 0 . 0 0 0}$ por $\mathbf{m m}$ cúbico, proteína $\mathrm{C}$ reactiva (PCR) y perfil biofísico (PB).

El $48 \%$ tenían entre 29 y 32 semanas al momento de la ruptura. El periodo de latencia promedio fue de 7 días. El síndrome de dificultad respiratoria neonatal se presentó en el $25 \%$ y fue responsable de 3 muertes, la infección neonatal precoz representó el $23 \%$.

En el 36,5\% se presentó infección materna que correspondió a corioamnionitis clínica en $28,8 \%$ de los casos y a endometritis en el $15 \%$ de ellos.

Para el diagnóstico precoz de corioamnionitis, la leucocitosis y PCR tuvieron una sensibilidad del 66,6\% y una especificidad del 75,7\%, mientras el PBF presentó una sensibilidad del $22,2 \%$ y una especificidad del $57,1 \%$, observando que la calificación de sus diferentes parámetros dependió de la experiencia del ecografista.

PALABRAS CLAVES: Ruptura prematura de membranas, manejo conservador, corioamnionitis, leucocitosis, proteína $\mathrm{C}$ reactiva, perfil biofísico.

\section{SUMMARY}

This is a descriptive-prospective study in 52 women with diagnose of membrane rupture between 26 an 35 weeks to whom an expectant management was performed. They were followed up for clinical signs of chorioamnionitis and laboratory findings of leucocitosis, changes on serum $\mathrm{C}$ reactive protein (PCR) and abnormal biophysical profile.

$48 \%$ of the patients were between 29 an 32 weeks of gestation at the moment of rupture. The average latency period was of 7 days. The respiratory distress syndrome was $25 \%$ and was responsible of 3 neonatal deaths. Early neonatal infection was $23 \%$.

Maternal infection was of $36,5 \%$ with $28,8 \%$ of chorioamnionitis and $15 \%$ of endometritis.

For early diagnosis of chorioamnionits, leucocitosis and PCR had a sensibility of $66,6 \%$ and a specificity of $75,7 \%$, while the biophysical profile had $22,2 \%$ and $51,7 \%$ respectively.

KEY WORDS: Premature rupture of membranes, expectant management, corioamnionitis, serum c reactive protein, leukocytosis, biophysical profile.

\section{Introducción}

La Ruptura Prematura de Membranas (RPM), es una patología de gran importancia clínica y epidemiológica, debido a la alta frecuencia de complicaciones maternofetales asociadas como: infección, prematuridad, síndrome de dificultad respiratoria del recién nacido,

\footnotetext{
* Docente, departamento de obstetricia y ginecología

** Residente, departamento de obstetricia y ginecología. Facultad de medicina, Universidad de Antioquia, Hospital San Vicente de Paúl, Medellín.
}

hemorragia intraventricular, enterocolitis necrotizante, displasia broncopulmonar, ductus arterioso permeable y retinopatía por prematurez.

Su manejo es controvertido, principalmente cuando ocurre antes de la semana 37 de gestación (Ruptura prematura de membranas pretérmino - RPMP),(1-3) y la decisión de terminar el embarazo inmediatamente, manejo activo, o en forma diferida, manejo conservador, es el resultado de sopesar los riesgos asociados a la prematuridad contra el riesgo séptico que aumenta con el tiempo de evolución de la ruptura.(4-6)

Para el manejo conservador algunos se atienen a criterios clínicos y otros le asignan un papel preponde- 
rante a exámenes como: hemoleucograma (HLG), proteína $C$ reactiva (PCR) y perfil biofísico fetal (PBF) para predecir infección en pacientes con RPMP. $(7,8,9)$

En la literatura nacional no se ha hecho una revisión sistemática de la validez de estos parámetros de laboratorio como predictores de infección en estos pacientes.

Nuestro objetivo es describir la evolución materna y neonatal de las pacientes con manejo conservador de RPMP entre 26 y 35 semanas de gestación, determinando frecuencia de infección materna, infección neonatal precoz, síndrome de membrana hialina y mortalidad perinatal. Además, evaluar la sensibilidad y especificidad como predictores de corioamnionitis de los siguientes parámetros: leucocitosis mayor de 20.000 por milímetro cúbico y aumento de la proteína $\mathrm{C}$ reactiva, y perfil biofísico menor de ocho.

\section{Materiales y métodos}

Se realizó un estudio descriptivo, prospectivo de 52 pacientes que ingresaron al Hospital San Vicente de Paúl (HUSVP) de Medellín - Colombia, con RPMP entre 26 y 35 semanas de gestación, en el período comprendido entre el $1^{\circ}$ de mayo de 1996 y el 30 de enero de 1998.

Dado que en estos casos la literatura informa frecuencia de corioamnionitis hasta del 30\%,10,11 el tamaño de la muestra fue calculado entre 38 y 41 pacientes, con un grado de confianza del $95 \%$ y un error del $5 \%$.

La edad gestacional se estableció por amenorrea confiable o ecografía expresándola en semanas cumplidas, verificando con el índice de Capurro para calcular la edad de los neonatos mayores de 28 semanas,(12) y el método de Ballard para los menores de dicha edad.(13)

Fueron criterios de exclusión: embarazo gemelar, alteraciones hipertensivas asociadas, patología renal materna, diabetes, isoinmunización, enfermedades del colágeno, inmunosupresión, malformaciones fetales mayores o retardo del crecimiento intrauterino.

El diagnóstico de RPMP se estableció por interrogatorio a la paciente, visualización directa de canal cervical por especuloscopia o hallazgos específicos de disminución marcada del líquido amniótico, definido por el índice ecográfico de PHELAN menor de cinco.(14-15) Si a pesar de los parámetros anteriores persistió la duda, se realizó una amnioinfusión dirigida por ecografía, con cinco centímetros cúbicos de índigo carmín en una solución al $2 \%$ diluida hasta $10 \mathrm{~cm}$ en suero salino fisiológico, observando la tinción de un apósito estéril previamente colocado en vagina.(16)

El tacto vaginal se realizó sólo a pacientes con trabajo de parto activo.

Establecido el diagnóstico se hizo el siguiente manejo:

1. Reposo relativo

2. Control de pulso y temperatura axilar cada cuatro horas

3. Hemoleucograma y velocidad de sedimentación globular cada 24 horas

4. Proteína $\mathrm{C}$ reactiva cuantitativa cada 24 horas

5. Citoquímico de orina, urocultivo, directo y gram de flujo vaginal, al ingreso

6. Perfil biofísico (PB) cada 24 horas
7. Betametasona $12 \mathrm{mg}$ intramuscular al ingreso y a las 24 horas, a todas las pacientes entre 26 y 34 semanas, si la prueba de Clements fue negativa o no se pudo realizar.(17-19)

8. Pacientes con trabajo de parto entre 26 y 34 semanas, se uteroinhibieron por vía endovenosa con Sulfato de Magnesio o Terbutalina, en la forma convencional,20 hasta 12 horas después de la última dosis de esteroides.

9. Fue motivo de terminación del embarazo la presencia de:

- Corioamnionitis clínica

- Aumento de los leucocitos hasta 20.000 o más junto con un aumento de la proteína $\mathrm{C}$ reactiva.

- Evidencia mediante Monitoreo Fetal Electrónico de sufrimiento fetal agudo

- Inicio espontáneo de actividad uterina después de 12 horas de la última dosis de betametasona.

- Perfil biofísico con un puntaje menor de ocho o ausencia de movimientos fetales.(21-23)

10. En caso de corioamnionitis clínica se ordenó antibioticoterapia con Gentamicina $4 \mathrm{mg} / \mathrm{kg} /$ día dividido en tres dosis vía intramuscular o intravenosa diluida en 100cc de suero salino fisiológico, asociada a Penicilina Cristalina 5 millones de unidades internacionales vía endovenosa cada seis horas o en su defecto Clindamicina $600 \mathrm{mg}$ vía endovenosa cada seis horas tres dosis.

11. En los partos por cesárea sin evidencia de infección, se usó antibioticoterapia profiláctica con una Cefalosporina de primera generación un gramo endovenoso una vez pinzado el cordón umbilical, repetido a las seis horas.

12. El manejo expectante se realizó hasta las 34 semanas cuando se terminó la gestación por cesárea o vía vaginal según las condiciones obstétricas.

13. La hospitalización posparto fue mínimo 72 horas.

14. Los neonatos se manejaron de acuerdo con los protocolos del Servicio de Neonatología del Hospital San Vicente de Paúl.

15. Luego del alta se hizo control a los 10 días, para evaluar la presencia de infección puerperal o neonatal.

Se hizo la siguiente definición de términos:

CORIOAMNIONITIS CLÍNICA: presencia de fiebre mayor de $37,8^{\circ} \mathrm{C}$, tomada en la axila, más dos de los siguientes criterios: minuto

Taquicardia materna mayor de 100 latidos por

- Taquicardia fetal mayor de 160 latidos por minuto

- Leucocitosis mayor o igual a 20.000 por milímetro cúbico

- Sensibilidad uterina

- Flujo vaginal fétido

Debe existir ausencia de otros focos de infección o del uso de fármacos que puedan alterar las cifras anotadas.

INFECCIÓN PUERPERAL: es aquella que se desarrolla en el tracto genital después del parto. Fue definida por el Joint Commitee on Maternal Welfare como "Temperatura de $38^{\circ} \mathrm{C}$ o más que aparece durante dos días en el transcurso de los 10 días que siguen al parto, excluyendo las primeras 24 horas, temperatura que es determinada por vía oral, mediante el empleo de una técnica estándar, por lo menos cuatro veces al día". 
SEPSIS NEONATAL PRECOZ: infección generalizada que ocurre durante las primeras 96 horas de vida con presencia de signos clínicos manifiestos y acompañada de aislamiento de un germen en el hemocultivo. Es causado por microorganismos adquiridos "in útero" o durante el paso por el canal del parto.

SINDROME DE DIFICULTAD RESPIRATORIA DEL NEONATO (ENFERMEDAD DE MEMBRANA HIALINA): definida por la presencia de los siguientes factores: comienzo temprano después del nacimiento, taquipnea con quejido espiratorio y retracciones inspiratorias, cianosis e hipoxia, y hallazgos típicos en la radiografía de tórax.24

SUFRIMIENTO FETAL: es una alteración del intercambio metabólico entre la madre y el feto que trae como consecuencia hipoxemia, acidosis e hipercapnia al igual que otros disturbios homeostáticos fetales. Se determinó mediante las alteraciones características en los patrones de monitoreo fetal electrónico. 25

MORTALIDAD PERINATAL: se refiere a las muertes de los productos de la concepción feto o recién nacido, que ocurre en el período perinatal, el cual está comprendido entre la semana 28 de gestación y los 7 días posparto.

PERFIL BIOFISICO: es un método de vigilancia fetal basado en la observación conjunta de cinco actividades biofísicas: movimientos respiratorios fetales, movimientos corporales gruesos, tono fetal, reactividad cardiaca fetal (monitoría sin estrés), y volumen del líquido amniótico. Se asigna un puntaje de dos cuando el parámetro está presente, y cero cuando está ausente, así el máximo puntaje es de 10 y el mínimo de 0 .

PERÍODO DE LATENCIA: es el tiempo transcurrido entre la ruptura de las membranas ovulares y el momento del parto.

En el análisis estadístico se utilizaron razones y proporciones para calcular frecuencia de presentación de los eventos. Para el cálculo de sensibilidad y especificidad de las pruebas de laboratorio (leucocitosis mayor de 20.000 , aumento en proteína $\mathrm{C}$ reactiva, y PB), se tomó como referencia el diagnóstico clínico de corioamnionitis, utilizando las pacientes infectadas para calcular sensibilidad y las no infectadas para especificidad, por medio de tablas de 2 por 2 .

Para leucocitosis y proteína $\mathrm{C}$ reactiva, el número de pacientes fue 52 (100\% de los casos estudiados), y para PB fue de 30 pacientes (58\% de los casos estudiados). Esta diferencia se debió a limitaciones técnicas o de tiempo en el Servicio de Urgencias de Obstetricia y Ginecología del HSVP.

\section{Resultados}

Maternos:

El $79 \%$ de las pacientes que ingresaron al estudio tenían entre 15 y 34 años de edad (figura No. 1), y el $80 \%$ eran multíparas (figura No. 2).

La edad gestacional promedio al momento de la RPMP fue de 31 semanas, con el $48 \%$ de las pacientes entre 29 y 32 semanas (figura No. 3). El período de latencia varió entre 1 y 43 días, con un promedio de 7 días.
El $56 \%$ de las pacientes tuvo parto vaginal.

En quince pacientes se hizo el diagnóstico de corioamnionitis clínica $(28,8 \%) 4$ de las cuales evolucionaron a endometritis. De otro lado 4 pacientes tuvieron endometritis sin corioamnionitis previa. Estas 19 pacientes con infección materna representan el 36,5\% de la población estudiada.

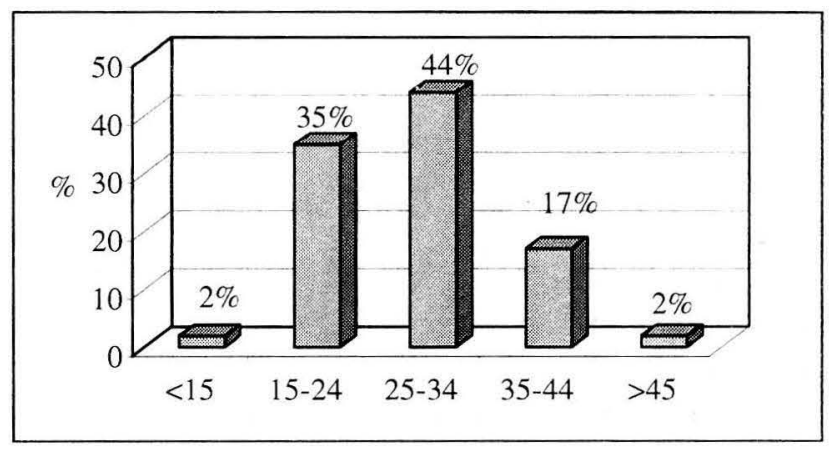

Figura 0021i01

EDAD DE LAS PACIENTES CON RUPTURA PREMATURA DE MEMBRANA PRETERMINO HUSVP. MEDELLIN, MAYO DE 1996 A ENERO DE 1998

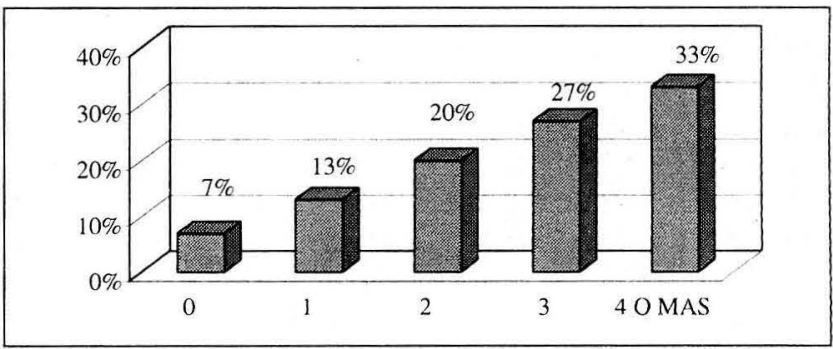

Figura $0021 \mathrm{i02}$

PARIDAD DE LAS PACIENTES CON RUPTURA PREMATURA DE MEMBRA PRETERMINO EN HUSVP. MEDELLIN, MAYO DE 1996 A ENERO DE 1998

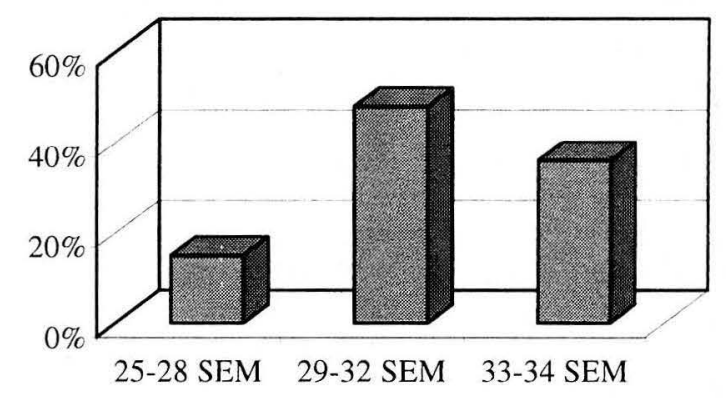

Figura 0021 i03

EDAD GESTACIONAL AL MOMENTO DE LA RUPTURA PREMATURA DE MEMBRANA PRETERMINO EN PACIENTES DEL HUSVP. MEDELLIN, MAYO DE 1996 A ENERO DE 1998 
La infección materna fue más frecuente después de 48 horas de ruptura (figura No. 5).

El $75 \%$ de las madres tuvo una estancia hospitalaria posparto menor de una semana (tabla No. 2).

\section{Neonatales:}

Hubo tres muertes neonatales que representan el $6 \%$ de la población estudiada. Todos tuvieron en común, parto por cesárea, peso menor de $1.000 \mathrm{~g}$ y síndrome de membrana hialina. Además, uno de ellos presentó sepsis neonatal precoz.

De los cinco neonatos que pesaron menos de $1.100 \mathrm{~g}$, dos sobrevivieron, pero uno sufrió hemorragia intraventricular grado I. En general el peso de los neonatos osciló entre 1.000 y $2.750 \mathrm{~g}$, con un promedio de $1.834 \mathrm{~g}$.

La frecuencia de síndrome de dificultad respiratoria fue del $25 \%$, y de infección neonatal precoz del $23 \%$ (tabla No.1). El $70 \%$ de los neonatos tuvo una hospitalización mayor de cuatro días (tabla No. 2).

La infección neonatal fue más frecuente después de 48 horas de ruptura (figura No. 4).

Con respecto a las pruebas diagnósticas encontramos:

Para el diagnóstico precoz de corioamnionitis, tanto la leucocitosis como la proteína $\mathrm{C}$ reactiva tuvieron una sensibilidad $66,6 \%$ y una especificidad del $75,7 \%$.

El perfil biofísico mostró una sensibilidad de $22,2 \%$ y una especificidad de $57,1 \%$ para el mismo propósito.

\section{Discusión}

No existe ninguna controversia cuando la RPMP se acompaña de sufrimiento fetal o corioamnionitis clínica, en cuyo caso la terminación del embarazo es la conducta indicada. Sin embargo, cuando ésta se produce sin signo alguno de trabajo de parto, corioamnionitis clínica o sufrimiento fetal, el resultado perinatal está sometido a un delicado equilibrio entre prematuridad e infección corioamniótica (sepsis materna o fetal). El tiempo juega entonces un papel preponderante y todos los esfuerzos médicos se dirigen a obtener una rápida madurez fetal con un mínimo de riesgo séptico.

Tabla 1

RESULTADO NEONATAL DE LOS PACIENTES CON RUPTURA PREMATURA DE MEMBRANAS PRETERMINO, HUSVP. MEDELLIN. MAYO DE 1996 A ENERO DE 1998

\begin{tabular}{|l|c|c|}
\hline RESULTADO NEONATAL & No. de casos & $\%$ \\
\hline Síndrome de dificultad & & \\
respiratoria & 13 & 25 \\
Infección neonatal precoz & 12 & 23 \\
Mortalidad neonatal & 3 & 6 \\
Parto vaginal & 29 & 56 \\
Parto por cesárea & 23 & 44 \\
\hline Peso promedio & 1834 gramos \\
\hline
\end{tabular}

Número total de pacientes: 52
El manejo conservador implica el establecimiento tanto de medidas generales (reposo, corticoesteroides, uteroinhibición o antibióticos) como medidas particulares dirigidas a la detección precoz de la infección corioamniótica. Así la prueba ideal anteparto debe ser altamente sensible y específica, ya que la baja sensibili-

Tabla 2

TIEMPO DE HOSPITALIZACION MATERNO Y NEONATAL DE LOS PACIENTES CON RUPTURA PREMATURA DE MEMBRANAS PRETERMINO. HUSVP. MEDELLIN. MAYO DE 1996 A ENERO DE 1998

\begin{tabular}{|l|cc|cc|}
\hline \multirow{2}{*}{$\begin{array}{c}\text { TIEMPO DE } \\
\text { HOSPITALIZACION }\end{array}$} & MADRE & \multicolumn{2}{|l|}{ NEONATO } \\
\cline { 2 - 5 } & $\begin{array}{c}\text { No. de } \\
\text { casos }\end{array}$ & $\begin{array}{l}\text { No. de } \\
\text { casos }\end{array}$ & \% \\
\hline Menor de 4 días & 10 & 19 & 16 & 30 \\
De 4 a 7 días & 29 & 56 & 18 & 35 \\
Mayor de 7 días & 13 & 25 & 18 & 35 \\
\hline
\end{tabular}

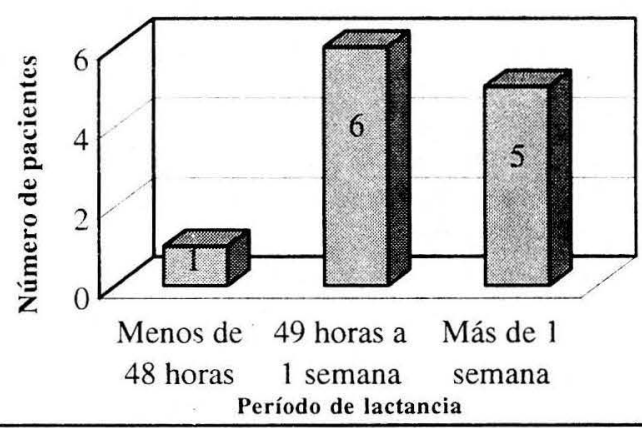

Figura $0021 \mathrm{i04}$

FRECUENCIA DE INFECCION NEONATAL PRECOZ CONTRA PERIODO DE LACTANCIA EN PACIENTES CON RUPTURA PREMATURA DE MEMBRANA PRETERMINO. HUSVP MEDELLIN MAYO DE 1996 A ENERO DE 1998

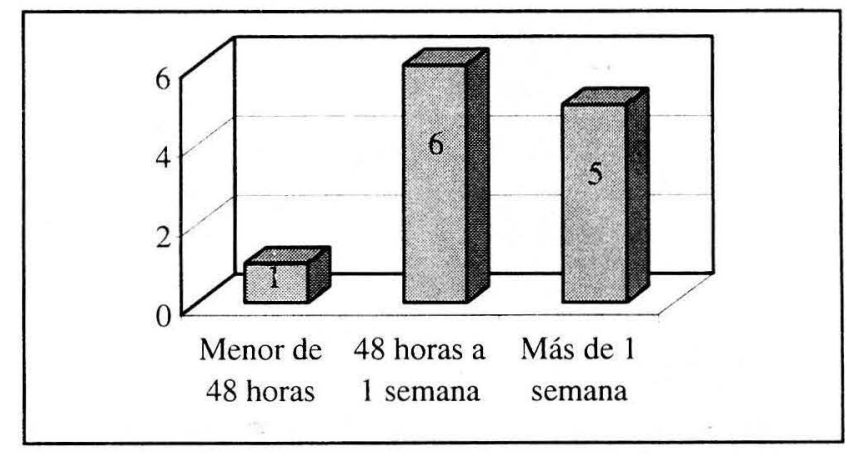

Figura 0021i05

FRECUENCIA DE INFECCION MATERNACONTRA PERIODO DE LACTANCIA PREMATURA DE MEMBRANAS PRETERMINO. HUSV. MEDELLIN. MAYO DE 1996 A ENERO DE 1998 
dad da como resultado muertes in útero no esperadas y la baja especificidad resulta en un alto número de intervenciones innecesarias, conllevando la posibilidad de iatrogenia fetal o materna.

Con el manejo actual de la ruptura prematura de membranas pretérmino que se hace en el HSVP se obtuvo en general un resultado perinatal aceptable, ya que el $77 \%$ de los neonatos no tuvieron infección, y el síndrome de dificultad respiratoria no fue tan frecuente $(25 \%)$, a pesar de que el $48 \%$ de las pacientes tuvieron ruptura prematura de membranas entre las 29 y 32 semanas de gestación. La frecuencia de sepsis neonatal precoz en el grupo estudiado fue comparable con el grupo total de neonatos pretérmino en el Servicio de Perinatología del HSVP de Medellín durante el mismo periodo de tiempo, y también con los hallazgos informados en la literatura médica mundial.26

La frecuencia de infección materna fue alta, 36,5\%, sin embargo, la respuesta al tratamiento con antibióticos fue rápida, con corta estancia hospitalaria, sin complicaciones, secuelas o mortalidad.

En nuestro estudio se usaron corticoesteroides en el $100 \%$ de los casos. Se obtuvo $25 \%$ de síndrome de dificultad respiratoria, representado en su gran mayoría por casos leves o moderados que no requirieron Unidad de Cuidados Intensivos, y con una mortalidad perinatal de sólo $6 \%$, conformada en su totalidad por fetos menores de $1.100 \mathrm{~g}$, en los cuales el factor determinante fue el bajo peso. Otros autores informan una frecuencia de mortalidad perinatal entre $14 \%$ y $31 \%$ para el manejo conservador de la ruptura prematura de membranas pretérmino, aún con el uso de esteroides para maduración.(27) En el Servicio de Perinatología del HSVP, se presentó una mortalidad perinatal total de $31,3 \%$ durante el mismo periodo de tiempo del estudio.

La sensibilidad y especificidad, como predictores de corioamnionitis, de la proteína $\mathrm{C}$ reactiva y la leucocitosis mayor de 20.000 por mm3 fueron comparables con los informados en la literatura. $(4,17)$

Con respecto al perfil biofísico menor de ocho como predictor de corioamnionitis, encontramos una baja sensibilidad y especificidad. Sin embargo, el menor número de pacientes utilizados para evaluar el $\mathrm{PB}$, no permite aprobar o rechazar este método diagnóstico.

Por lo tanto, concluimos que a pesar de los buenos resultados del PB como predictor de corioamnionitis informados por otros autores,(6) esta herramienta diagnóstica debe ser mejor evaluada en nuestro medio, con nuevos trabajos prospectivos en el tiempo, y estandarizada para permitir el conocimiento y adecuado manejo de esta prueba.

Se han hecho metaanálisis que muestran que la morbilidad infecciosa del neonato es menor cuando se usan antibióticos en el manejo expectante de la ruptura prematura de membranas pretérmino.(28-30) Sin embargo, es necesario valorar el alto costo de su uso y el riesgo de resistencia de las bacterias que podría presentarse a largo plazo(31) contra el buen resultado que se obtuvo en este trabajo con el manejo actual sin antibióticos que se realiza en nuestro hospital. Se hace necesario llevar a cabo un trabajo de investigación en nuestro medio, con el mismo manejo de la ruptura prematura de membranas, más el uso de antibióticos, para evaluar si la disminución de la morbilidad infecciosa, si se logra, tiene suficiente peso para justificar los costos y los riesgos que implicaría el uso de estos.

\section{Agradecimientos}

Doctor Rafael J. Manotas C., Docente del Centro de Investigaciones Médicas. Facultad de Medicina. Universidad de Antioquia.

Doctor Fernando Montoya M., Docente del Centro de Investigaciones Médicas. Facultad de Medicina. Universidad de Antioquia.

\section{BIBLIOGRAFIA}

1. Bada HS, Alojipan LC, Andrews BF: Premature rupture of membranes and its effect on the newborn. Pediatr Clin North Am. 1977; 24:491.

2. Wolf RL, Olinsky A: Prolonged rupture of fetal membranes and neonatal infections. S Afr Med J. 1976; 50:574.

3. Druzin ML, Toth M, Ledger WJ: Nonintervention in premature rupture of the amniotic membranes. Surg Gynecol Obstet. 1986; 163:5.

4. Davidson KM: Detección de la ruptura prematura de membranas. Clinic Obstet Gynecol. 1991; 4:685.

5. Manning FA, Platt LD, Sipos L. Antepartum fetal evaluation. Development of a fetal biophysical profile score 1980; Am J Obstet Gynecol. 136: 787-795.

6. Vintzileos AM, Campbell WA, Nochimson DJ, Conolly ME: The fetal biphysical profile in patients with premature rupture of the membranes-an early predictor of fetal infection. Am J Obstet Gynecol. $1985 ; 152: 510$.

7. Fayez JA, Hasan AA, Jonas HS, et al: Management of premature rupture of the membranes. Obstet Gynecol. 1978; 52:17.

8. Cox S, Willow ML, Levento KJ: The natural history of the preterm ruptured membranes: What to expect of expectant management. Obstet Gynecol. 1988; 71:558.

9. Vintzileos AM, Campbell WA, Ingardia CJ, Nochimson DJ: The fetal biophysical profile and its predictive value. Obstet Gynecol. 1983; 62:271.
10. Allen SR: Epidemiología de la rotura prematura de las membranas fetales. Clin Obst y Ginec 1991; 4:657.

11. Gibbs RS, Blanco JD: Premature rupture of the membranes. Obstet Gynecol. 1982; 60:671-9.

12. Capurro, H. Estimación de la edad gestacional por examen del neonato. En Aspectos perinatales del parto prematuro. Althabe D. y Schwartz R. Ed. Librería el Ateneo. Buenos Aires. 1978;77-81.

13. Ballard JL, et al. A simplified assessment of gestational age. Pediatr Res. 1977; 11: 374.

14. Romero R, Gomez R, Galasso M, Salafia C, Yoon BH, Behnke E, Cotton D: Oligohydramnios a risk factor for infection in term premature rupture of membranes? Ultrasound in Obstet and Gynecology.1994; 4:95.

15. Phelan, J.P, et al. Amniotic fluid volume assessment using the fourquadrant technique in the pregnancy. J. Reprod. Med. 1987; 32:540.

16. Gunn GC, Mishel DR, Morton DG: Premature rupture of the fetal membranes: A review. Am J Obstet Gynecol. 1970. 106:469.

17. Nelson LH, Anderson RL, O'Sea M: Expectant management of preterm premature rupture of membranes. Am J Obstet Gynecol. 1994; 171:351.

18. Liggins GC, Howie RN: The prevention of RDS by maternal steroid THERAPY. In Guck L (ed): Modern Perinatal Medicin. Chicago, Year Book 1994 
19. Jonathan M Klein: Neonatal morbidity and mortality secondary to premature rupture of membranes. Obstet Gynecol Clinic of North Am. 1992; 19:265.

20. Arias F: Guía practica para el embarazo y el parto de alto riesgo. $2 \mathrm{a}$ ed. St. Louis. M. Mosby/Doyma,. 1994; 101-114.

21. Vintzileos AM, Campbell WA, Nochimson DJ, Weimbaun PJ: The use of the nonstress test in patients with premature rupture of the membranes. Am J Obstet Gynecol. 1986; 155:149.

22. Vintzileos AM, Campbell WA, Nochimson DJ, Weimbaun PJ: Fetal breathing as a predictor of infection in premature rupture of the membranes. Obstet Gynecol. 1986; 67:813.

23. Goldstein $\mathrm{Y}$, Romero R, Merrill S, Wan M, O'connor TZ, Mazor M, et al: Fetal body and breathing movements as predictor of intraamniotic infection in preterm premature rupture of membranes. Am J Obstet Gynecol. 1988; 159:363.

24. Daikoku NH, Kaltreider F, Johnson TRB, et al: Premature rupture of membranes and preterm labor: Neonatal infection and perinatal mortality risks. Obstet Gynecol. 1981; 58:417.

25. Devoe JD, Castillo RA, Shrline DM: The Non Stress Test as a diagnostic test: A critical reappraisal. Am J. Obstet Gynecol. 1985; 152:1047-1053.
26. Bedyoun SN, Yasin SY: Premature rupture of the membranes before 28 weeks. Conservative management. Am J. Obstet Gynecol. 1986;155:47.

27. Leitich H, Egartes C, Reissemberber K. Concomitant use of glucocorticids: A comparison of two metaanalisyses on antibiotic treatment in preterm premature rupture of membranes. Am J Obstet Gynecol. 1998; 178:899-908.

28. Ananth Cande, Guise JM, Thorp John. Utility of antibiotic therapy in preterm premature rupture of membranes: a meta: analysis. Obstet Gynecol Surv. 1996; 51:5.

29. Mercer Brian, Arheart K. Antimicrobial therapy in expectant management of preterm premature rupture of membranes. Lancet $1995 ; 346: 1271-1279$.

30. Egarter Christian, Leitic $\mathrm{H}$, et al. Antibiotic treatment in preterm premature rupture of membranes and neonatal morbidity: a metanalysis. Am J Obstet Gynecol. 1996; 174:589-97.

31. McDuffie RS, McGregor JA, Gibbs RS. Adverse perinatal outcome and resistant enterobacteriaceae after antibiotic usage for premature rupture of the membranes and group B streptococcus carriage. Obstet Gynecol. 1993; 82:487-9.

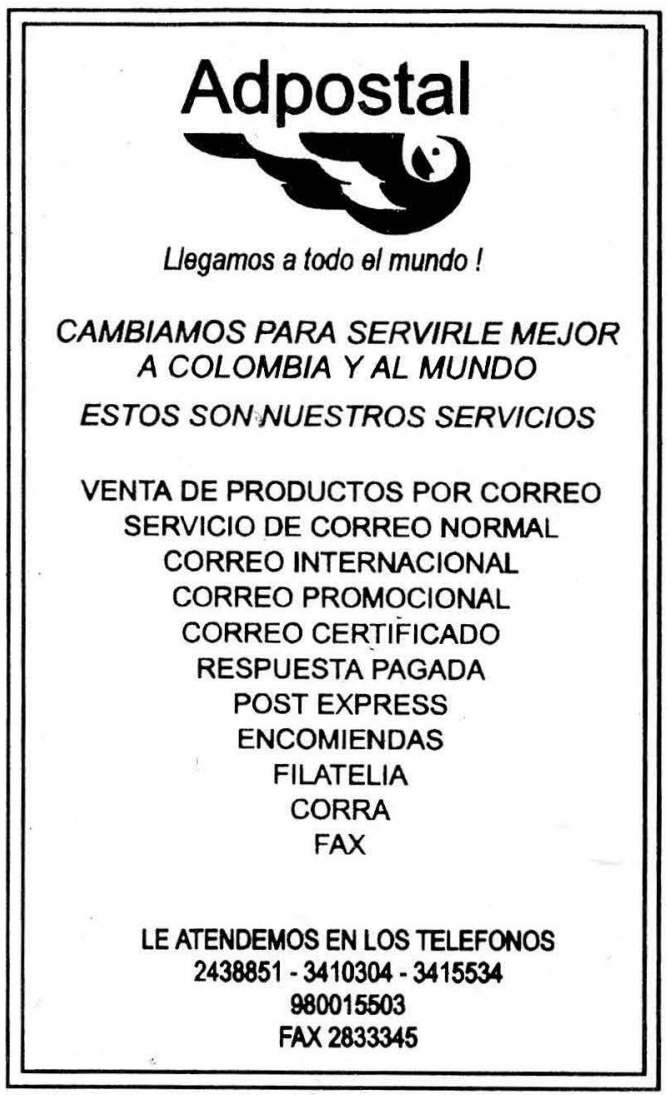

